\title{
Copycat Burkholderia make a move
}

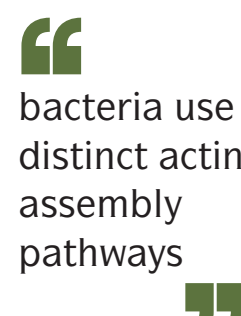

Burkholderia pseudomallei and Burkholderia mallei are intracellular pathogens that can cause human infections, whereas their close relative, Burkholderia thailandensis, is non-pathogenic. This difference in virulence has been puzzling, as these species share key virulence factors.

Now, Welch and colleagues show that mechanistic differences in the way these strains manipulate host actin-polymerizing proteins to drive actin-based motility influence the outcome of infection.

Burkholderia spp. invade host cells and exploit their actin assembly pathways for cell-to-cell spread and dissemination. The trimeric autotransporter Burkholderia intracellular motility A (BimA) is

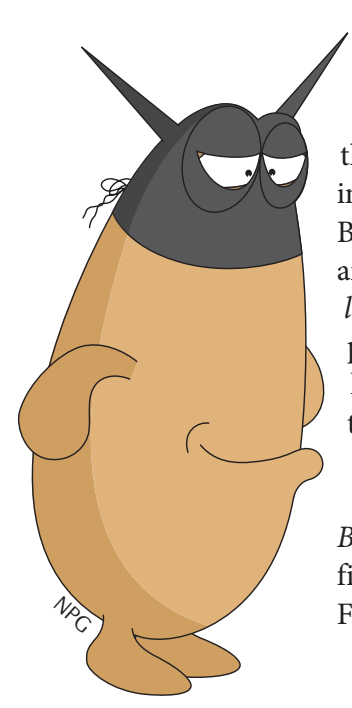
required for actin-based motility; however, the molecular mechanisms underlying this process in the three species were unclear. To investigate this, the authors purified BimA proteins from each species and showed that BimA from $B$. thai landensis functions as a nucleationpromoting factor that activates the host ARP2/3 complex to facilitate the assembly of branched actin filament networks. By contrast, BimA from B. pseudomallei and B. mallei generated unbranched filaments, independently of ARP2/3. Furthermore, BimA from the two pathogenic strains attached to the fast-growing barbed end of actin filaments, increased elongation rates of bundled filaments and exhibited anti-capping activity, suggesting that these BimA proteins mimic the host actin polymerase ENA/VASP.

B. pseudomallei and B. mallei BimA contain three and one $\mathrm{WH} 2$ domains, respectively, which have been implicated in actin polymerization by ENA/VASP. In agreement with this, the authors showed that the BimA $\mathrm{WH} 2$ domains from these two species bind to monomeric actin and that this interaction is required for their actin polymerization activities. Next, they replaced the endogenous $\operatorname{bim} A$ gene in $B$. thailandensis with that from B. pseudomallei or B. mallei. Using confocal microscopy, they showed that the actin-tail structure produced by wild-type $B$. thailandensis in infected cells was curved and consisted of a dense actin network; by contrast, $B$. thailandensis strains expressing BimA from B. pseudomallei or B. mallei generated longer, straighter tails that comprised bundled actin filaments. Time-lapse studies revealed that, although all strains moved with similar velocity, motility efficiency was reduced in strains with curved, short tails, suggesting that these bacteria use distinct actin assembly pathways to fine-tune motility.
Finally, the authors tested how the different actin polymerization mechanisms affect bacterial-mediated host cell fusion, which is essential for virulence of Burkholderia spp., and showed that a $B$. thailandensis strain expressing BimA from B. mallei exhibited reduced fusion efficiency compared with $B$. thailandensis strains expressing BimA from B. pseudomallei or from B. thailandensis; this reduction correlated with both decreased actin tail formation and reduced motility initiation. Notably, by mutating different WH2 motifs, the authors showed that BimA from B. pseudomallei requires two WH2 motifs for actin-based motility and fusion, whereas a single WH2 motif in BimA from B. mallei is sufficient to mediate these processes.

In summary, the results of this study suggest that closely related Burkholderia species developed divergent actin polymerization strategies, possibly to adapt to their specific hosts. The authors propose that these distinct mechanisms are key to the evolution of virulence, as both pathogenic strains mimic host ENA/VASP proteins.

Andrea Du Toit

ORIGINAL RESEARCH PAPER Benanti, E. L., Nguyen, C. M. \& Welch, M. D. Virulent Burkholderia species mimic host actin polymerases to drive actin-based motility. Cell 161, 348-360 (2015) 